\title{
FORMACIÓN PERMANENTE Y AUTOPERCEPCIÓN DE COMPETENCIA: UN ESTUDIO CON ENTRENADORES DE TENIS ${ }^{1}$
}

\author{
CONTINUING EDUCATION AND SELF-PERCEPTION OF COMPETENCE: A STUDY WITH \\ TENNIS COACHES
}

\section{FORMAÇÃO CONTINUADA E AUTOPERCEPÇÃO DE COMPETÊNCIA: UM ESTUDO COM TREINADORES DE TÊNIS}

\author{
Caio Corrêa Cortela ${ }^{1}$, Michel Milistetd ${ }^{2}$, Jorge Both ${ }^{3}$, Larissa Rafaela Galatti ${ }^{4}$, Miguel Crespo ${ }^{5}$, \\ Carlos Adelar Abaide Balbinotti ${ }^{6}$ \\ caio.tenis@yahoo.com.br; michel.milistetd@ufsc.br; jorgeboth@yahoo.com.br; \\ larissa.galatti@fca.unicamp.br; miguel.crespo@itftennis.com; carlos.balbinotti@ufrgs.br \\ ${ }^{1}$ Federação Paranaense de Tênis, Brasil 2 Universidade Federal de Santa Catarina, Brasil \\ ${ }^{3}$ Universidade Estadual de Londrina, Brasil ${ }^{4}$ Universidade Estadual de Campinas, Brasil \\ ${ }^{5}$ Federação Internacional de Tênis, Reino Unido \\ ${ }^{6}$ Universidade Federal do Rio Grande do Sul, Brasil
}

Envio Original: 2019-04-15 Reenviado: 2019-09-24 Aceptado: 2019-10-29

Publicado en versión en español: 2020-04-28

Doi: https://doi.org/10.15517/pensarmov.v18i1.41586

\begin{abstract}
Resumen
Este estudio tuvo como objetivo asociar la formación permanente de entrenadores con la autopercepción y la importancia atribuida al constructo - competencia profesional. La muestra fueron 69 entrenadores de tenis, que participaron de actividades de capacitación promovidas por la Federación Paranaense de Tenis, estando constituida mayormente por profesionales: con más de ocho años de experiencia profesional; con experiencia como practicante; y graduados en Educación Física. Para la recolección de datos se utilizó una ficha con variables socio demográficas y la Escala de Auto Percepción de Competencia. Los resultados indicaron que la
\end{abstract}

\footnotetext{
1 Versión traducida al español. Original en portugués disponible en esta misma revista. Cortela, C., Milistetd, M., Both, J., Galatti,L., Crespo, M., y Balbinotti, C. (2019). Formação continuada e autopercepção de competência: um estudo com treinadores de tênis. Pensar en Movimiento: Revista de Ciencias del Ejercicio y la Salud, $17(2)$, e36948. https://doi.org/10.15517/pensarmov.v17i2.36948
} 
participación en actividades/cursos no tuvo asociación significativa con la autopercepción de competencia de los entrenadores para los Conocimientos Profesionales. Para la dimensión de Habilidades Profesionales, involucrarse en actividades de capacitación se relacionó significativamente con la autopercepción de dominio, con énfasis en las habilidades relacionadas con el Planeamiento y Gestión Deportiva, y Evaluación Deportiva, habilidades consideradas centrales en el ejercicio profesional de los entrenadores. La importancia atribuida a los Conocimientos y Habilidades Profesionales fue elevada, siendo siempre superior a la autopercepción de dominio.

Palabras clave: conocimiento profesional, competencias profesionales, formación de entrenadores, desarrollo profesional.

\begin{abstract}
The aim of this study was to associate the coaches' continuing education and self-perception and the importance attributed to the construct of coaches' professional competence. The sample was 69 tennis coaches, whom have participated in professional training actions promoted by the Paraná Tennis Federation. The characteristics of participants were: more than eight years of professional experience; experience as a tennis player; and graduate level in Physical Education. A chart containing the sociodemographic variables and the Competence Self Perception Scale were used on data collection. The results indicated that participation in workshops / courses did not demonstrate a significant association with the self-perception of competencies for the Professional Knowledge dimension. For the Professional Skills dimension, involvement in these actions was significantly related to self-perception of mastery, with emphasis on skills related to Sports Planning and Sports Management and, Sports Assessment, skills considered as pivotal to the professional coaching intervention. The importance attached to Knowledge and Professional skills was high, always being higher than the self-perception of domain.
\end{abstract}

Keywords: professional knowledge, professional skills, coaches, coach education, coach development.

\title{
Resumo
}

Este estudo teve como objetivo associar a formação continuada dos treinadores com a autopercepção e a importância atribuída ao constructo - competência profissional. A amostra foi composta por 69 treinadores de tênis, participantes de ações de capacitação promovidas pela Federação Paranaense de Tênis constituída, majoritariamente, por profissionais: com mais de oito anos de experiência profissional; com experiência como praticante; e graduados em 
Educação Física. Para recolha de dados utilizou-se uma ficha contendo as variáveis sociodemográficas e a Escala de Autopercepção de Competência. Os resultados indicaram que a participação em atividades/cursos não demonstrou associação significativa com a autopercepção de competência dos treinadores para os Conhecimentos Profissionais. Para a dimensão Habilidades Profissionais, o envolvimento nessas ações relacionou-se significativamente com a autopercepção de domínio, com destaque para as habilidades relacionadas ao Planejamento e Gestão Esportiva, e Avaliação Esportiva, habilidades consideradas centrais no exercício profissional do treinador. A importância atribuída aos Conhecimentos e Habilidades Profissionais foi elevada, sendo sempre superior à autopercepção de domínio.

Palavras-Chave: conhecimento profissional, competências profissionais, formação de treinadores, desenvolvimento profissional.

\section{Introducción}

La valorización de la actividad del entrenador deportivo se encuentra en el período más dinámico de su existencia. Las demandas de esta profesión evolucionaron del simple dominio de contenidos técnico/tácticos de una modalidad, a la necesidad de demostrar competencia en diversas áreas, tales como la capacidad de comunicarse con calidad, actuar en diferentes ambientes, reflexionar sobre su propia práctica, entre otros dominios (Milistetd, Galatti, Tozetto, Collet y Nascimento, 2017).

A pesar de la creciente complejidad del coaching deportivo en el siglo XXI y la consecuente exigencia de calificación en la intervención del entrenador, el reconocimiento del status profesional en esta área sigue siendo controversial (Taylor y Garrat, 2013). En muchos países la actuación como entrenador es realizada de forma voluntaria, principalmente cuando el contexto es de formación o participación deportiva (International Council for Coaching Excellence [ICCE], 2013). Sin embargo, en el deporte de rendimiento, el número de voluntarios es menor, actuando principalmente, entrenadores remunerados económicamente ya sea en tiempo parcial o por el tiempo completo (Duffy, Petrovic y Crespo, 2010).

Esta diversidad de entornos operativos, combinada con la necesidad de una intervención cada vez más calificada, demanda mejoras en los procesos formativos de los entrenadores deportivos (ICCE, 2013). Dentro de los principales cambios presentados en la literatura sobre la formación de estos profesionales es posible identificar un cambio en los paradigmas educativos en diferentes países (Ciampolini, Milistetd, Rynne, Brasil y Nascimento, 2019), el reconocimiento de la universidad como un medio formativo (Milistetd et al., 2017) y la 
concepción de que la preparación del entrenador es un proceso continuo que debe ocurrir a lo largo de sus carreras (Trudel y Gilbert, 2013).

Específicamente en Brasil, donde el entrenador deportivo es reconocido en todos sus contextos de actuación como un profesional (Gobierno de Brasil, 1998), ocurre el proceso de preparación formal, sobretodo en un ambiente universitario, por medio de la titulación como bachillerato (en Brasil) en Educación Física. Sin embargo, se ha demostrado que la formación inicial se ha limitado al desarrollo de conocimientos generales, con poco énfasis en las competencias esenciales para la intervención de entrenador deportivo, tales como: el establecimiento de una filosofía de trabajo; estrategias de comunicación y resolución de problemas, y la evaluación de la propia práctica (Milistetd et al., 2017).

En vista de esto, la formación permanente o continua asume un papel central en el desarrollo de esos profesionales (Rodrigues, Paes y Souza Neto, 2016). La participación en diferentes oportunidades de aprendizaje demuestra impactar directamente la precepción y la importancia atribuida por los entrenadores a los conocimientos y competencias profesionales. De modo general, los resultados de estudios que investigaron el papel desempeñado por la formación inicial/permanente y de experiencia apuntan a que las mismas influencian: en la preferencia por contenidos específicos relacionados con el conocimiento profesional (Egerland, Nascimento y Both, 2009a; Egerland, Nascimento y Both, 2010); en la importancia que se le atribuye a las competencias profesionales (Egerland et al., 2009a; Egerland et al., 2010); y en la autopercepción de competencia profesional (Egerland, Salles y Baldi, 2014). De esta manera, y con base en lo descrito previamente, el presente estudio tuvo como objetivo principal identificar la existencia de posibles asociaciones entre la formación permanente de entrenadores de tenis y la autopercepción e importancia atribuida al constructo - competencia profesional. De forma específica se buscó relacionar la autopercepción de competencias de esos profesionales con la importancia atribuida a los aspectos que componen la matriz de análisis de la competencia profesional.

\section{MÉTODOLOGÍA}

Tipo de Investigación y Muestra

El estudio se caracterizó como descriptivo-exploratorio, de corte transversal y de abordaje cuantitativo (Silva, Minatto, Fares y Santos, 2011). La muestra estuvo conformada por entrenadores de tenis que participaron en actividades de formación permanente desarrolladas 
por la Federación Paranaense de Tenis (FPT). De esta forma, participaron en el estudio 69 entrenadores (32,6 $\pm 9,3$ años de edad) de ambos sexos (64 hombres y 5 mujeres).

La mayor parte del grupo investigado poseía las siguientes características: socialización pre-profesional como 'junta bolas' (persona que recoge pelotas de tenis usadas) (46\%); y nivel técnico compatible con primera y segunda clase en el ranking FPT, en una escala de ocho clases (56\%). En lo que se refiere a la formación inicial y permanente, se observó que el 23\% de los entrenadores cursaban el bachillerato (en Brasil) en Educación Física al momento de recolección de los datos, mientras que el 51\% indicaron estar ya graduados en esa área. La mayoría (81\%) indicaron haber participado en actividades/cursos de formación permanente en los últimos cinco años. De estos, el 52\% estuvieron presentes en cuatro eventos o más. Finalmente, se destaca que el $51 \%$ de la muestra ejercen como entrenadores hace más de ocho años.

\section{Instrumentos}

Los instrumentos utilizados para la recolección de datos fueron: una ficha para obtener las variables sociodemográficas (edad, sexo, experiencia como practicante y entrenador, formación inicial y permanente); y la Escala de Autopercepción de Competencia (EAPC) de entrenadores deportivos, modificada para la realidad brasileña (Egerland, et al., 2010). La versión modificada de la EAPC es constituida por 78 preguntas cerradas, relacionadas con la autopercepción de competencia y la importancia atribuida, siendo organizada en una estructura bidimensional, compuesta por las dimensiones Conocimientos Profesionales y Habilidades Profesionales. La dimensión Conocimiento Profesional está compuesta por 46 ítems, pertenecientes a cuatro categorías: Gestión y Legislación del Deporte; Biodinámica; Aspectos Psico-socio-culturales del Deporte; y Teoría y Metodología del Entrenamiento. En esta misma dirección, la dimensión Habilidades Profesionales está constituida por 32 ítems subdivididos en cuatro áreas: Evaluación en el Deporte; Comunicación e Integración; Planeamiento y Gestión Deportiva; Autorreflexión y Actualización Profesional. Por medio de una escala tipo Likert de cinco puntos, los entrenadores indicaron el grado de dominio ( $1=$ no dominio y $5=$ muy buen dominio) e importancia atribuida ( $1=$ ninguna importancia y $5=$ mucha importancia), a los ítems presentados.

La adaptación transcultural del instrumento ocurrió en tres etapas. Primeramente se realizó la traducción y adecuación del instrumento al idioma portugués brasileño. En la segunda etapa se buscó evaluar la claridad y la objetividad del idioma, con 26 entrenadores participando de este proceso, quienes mostraron índices de consenso iguales o superiores al $80 \%$, en la relación dimensiones e ítems. Después de estos procedimientos se realizó una evaluación de 
reproducibilidad del instrumento con 50 entrenadores, y en este proceso todas las preguntas mostraron índices aceptables de concordancia verificándose valores superiores a 0,75 (Egerland et al., 2010).

\section{Procedimientos de recolección}

La recolección de datos se dio de manera presencial y con el consentimiento del FPT, durante la ejecución de tres actividades de formación permanente organizadas por la institución. Estas actividades se llevaron a cabo en dos clubes y en una academia de tenis, localizados en tres regiones distintas (Región Metropolitana de Curitiba, Oeste Paranaense y Norte Paranaense) del estado de Paraná - Brasil.

Después de explicar los objetivos de la investigación, los entrenadores fueron invitados a participar del estudio. A los profesionales que mostraron interés en colaborar con la investigación, se les entregó el Formulario de Consentimiento Informado y los instrumentos que debían responder.

\section{Análisis Estadístico}

Al finalizar el período de recolección, los datos obtenidos fueron transcritos a una plantilla del software Microsoft Excel, para Windows, específicamente elaborada para esa finalidad. Para categorizar los datos referentes a los conocimientos y habilidades profesionales, se utilizó una versión adaptada de ecuación ponderada descrita por Lemos (2007). Valores iguales y debajo de cero, en una escala de variabilidad de -100 a +100 , encontrados para la competencia profesional e importancia atribuida, se clasificaron respectivamente como: no domina y no importante. Por otro lado, valores iguales o superiores a 0,1 fueron categorizados como: Domina, para competencia profesional; e importante, para importancia atribuida.

Posteriormente, en el análisis estadístico de los datos fueron realizados cálculos de frecuencia y estadística descriptiva. Para comparaciones entre los grupos compuestos por entrenadores que frecuentaban y no frecuentaban actividades de formación permanente en los últimos cinco años, en lo que respecta a las dimensiones Conocimiento y habilidades Profesionales, y sus subcategorías, se empleó la prueba de Chi-cuadrado. En los casos en que las frecuencias esperadas en una de las celdas de las tablas $(2 \times 2)$ fueron menores de cinco, se optó por utilizar la prueba exacta de Fisher. Por su parte, para las comparaciones entre los resultados mostrados por los grupos de entrenadores para las variables, competencia percibida e importancia atribuida, se utilizó el test McNemar. Para todos los análisis fue pre-establecido un nivel de significancia con $p \leq 0,05$. 


\section{RESULTADOS}

Con respecto al análisis del constructo de habilidades profesionales, la muestra de entrenadores reportó un bajo nivel de dominio solo en el indicador de Gestión Deportiva y Legislación (37.7\%). Para la mayor parte de las dimensiones y categorías analizadas los valores encontrados fueron considerados razonables: Conocimientos $(58,0 \%)$ y Habilidades Profesionales (63,8\%); Evaluación Global de la Competencia Profesional (52,2\%); Evaluación en el Deporte (62,3\%); Biodinámica del Deporte (66,7\%); y Autorreflexión y Actualización profesional en el Deporte $(68,1 \%)$. Por último, altos índices de competencia profesional fueron evidenciados en los indicadores: Planeamiento y Gestión Deportiva (71,0\%); Comunicación e Integración (73,9\%); Psico-Sócio-Culturales del Deporte (76,8\%); Teoría e Metodología del Entrenamiento Deportivo (82,6\%) (Tabla 1).

\section{Tabla 1.}

Asociación entre autopercepción de dominio de competencia profesional y realización de cursos de formación permanente en entrenadores de tenis.

\begin{tabular}{|c|c|c|c|}
\hline Formación Permanente & Competenci & percibida & \\
\hline DIMENSIÓN Conocimientos Profesionales & No Domina & Domina & $\mathbf{p}$ \\
\hline Evaluación Global - Conocimientos Profesionales ${ }^{1}$ & & & 0,34 \\
\hline No asistió & $7(53,8 \%)$ & $6(46,2 \%)$ & \\
\hline Asistió & $22(39,3 \%)$ & $34(60,7 \%)$ & \\
\hline Total & $29(42,0 \%)$ & $40(58,0 \%)$ & \\
\hline Gestión y Legislación del Deporte ${ }^{2}$ & & & 0,06 \\
\hline No asistió & $11(84,6 \%)$ & $2(15,4 \%)$ & \\
\hline Asistió & $32(57,1 \%)$ & $24(42,9 \%)$ & \\
\hline Total & $43(62,3 \%)$ & $26(37,7 \%)$ & \\
\hline Biodinámica del Deporte ${ }^{2}$ & & & 0,55 \\
\hline No asistió & $4(30,8 \%)$ & $9(69,2 \%)$ & \\
\hline Asistió & $19(33,9 \%)$ & $37(66,1 \%)$ & \\
\hline Total & $23(33,3 \%)$ & $46(66,7 \%)$ & \\
\hline Psico-Socio-Culturales del Deporte ${ }^{2}$ & & & 0,35 \\
\hline No asistió & $4(30,8 \%)$ & $9(69,2 \%)$ & \\
\hline Asistió & $12(21,4 \%)$ & $44(78,6 \%)$ & \\
\hline Total & $16(23,2 \%)$ & $53(76,8 \%)$ & \\
\hline Teoría y Metodología del Entrenamiento Deportivo² & & & 0,16 \\
\hline No asistió & $4(30,8 \%)$ & $9(69,2 \%)$ & \\
\hline Asistió & $8(14,3 \%)$ & $48(85,7 \%)$ & \\
\hline Total & $12(17,4 \%)$ & $57(82,6 \%)$ & \\
\hline
\end{tabular}




\begin{tabular}{|c|c|c|c|}
\hline DIMENSIÓN - Habilidades Profesionales & No Domina & Domina & $\mathbf{p}$ \\
\hline Evaluación Global - Habilidades Profesionales $^{2}$ & & & $0,04^{*}$ \\
\hline No asistió & $8(61,5 \%)$ & $5(38,5 \%)$ & \\
\hline Asistió & $17(30,4 \%)$ & $39(69,6 \%)$ & \\
\hline Total & $25(36,2 \%)$ & $44(63,8 \%)$ & \\
\hline Planeamiento y Gestión Deportiva² & & & $<0,01^{*}$ \\
\hline No asistió & $8(61,5 \%)$ & $5(38,5 \%)$ & \\
\hline Asistió & $12(21,4 \%)$ & $44(78,6 \%)$ & \\
\hline Total & $20(29,0 \%)$ & $49(71,0 \%)$ & \\
\hline Evaluación en Deporte ${ }^{2}$ & & & $0,05^{*}$ \\
\hline No asistió & $8(61,5 \%)$ & $5(38,5 \%)$ & \\
\hline Asistió & $18(32,1 \%)$ & $38(67,9 \%)$ & \\
\hline Total & $26(37,7 \%)$ & $43(62,3 \%)$ & \\
\hline Comunicación e Integración ${ }^{2}$ & & & 0,07 \\
\hline No asistió & $6(46,2 \%)$ & $7(53,8 \%)$ & \\
\hline Asistió & $12(21,4 \%)$ & $44(78,6 \%)$ & \\
\hline Total & $18(26,1 \%)$ & $51(73,9 \%)$ & \\
\hline \multicolumn{2}{|c|}{ Autorreflexión y Actualización Profesional en Deporte ${ }^{2}$} & & 0,06 \\
\hline No asistió & $7(53,8 \%)$ & $6(46,2 \%)$ & \\
\hline Asistió & $15(26,8 \%)$ & $41(73,2 \%)$ & \\
\hline Total & $22(31,9 \%)$ & $47(68,1 \%)$ & \\
\hline Evaluación Global & No Domina & Domina & $\mathbf{p}$ \\
\hline Competencia Percibida $^{1}$ & & & 0,09 \\
\hline No asistió & $9(69,2 \%)$ & $4(30,8 \%)$ & \\
\hline Asistió & $24(42,9 \%)$ & $32(57,1 \%)$ & \\
\hline Total & $33(47,8 \%)$ & $36(52,2 \%)$ & \\
\hline
\end{tabular}

Legenda: (1) Chi-cuadrado; (2) Exacto de Fisher; $\left({ }^{*}\right)$ Diferencia Significativa.

Fuente: elaborada por los autores.

Al analizar la relación existente entre formación permanente y competencia profesional (Tabla 1), se observó que los entrenadores que asistieron a cursos de formación en los últimos cinco años mostraron índices más elevados de dominio en los indicadores Planeamiento y Gestión Deportiva $(p<0,01)$, Evaluación en Deporte $(p=0,05)$ y en la dimensión de Habilidades Profesionales $(p=0,04)$.

Sobre la importancia atribuida por los entrenadores a las unidades que componen el constructo de competencia profesional, se observó que todos los indicadores, dimensiones y evaluación global mostraron elevados índices de importancia (entre 79,7\% y 94,2\%) (Tabla 2). 
Tabla 2.

Asociación entre la importancia atribuida al constructo de competencia profesional y la realización de actividades/cursos de formación permanente en entrenadores de tenis.

\begin{tabular}{|c|c|c|c|}
\hline Formación Permanente & Importanci & atribuida & \\
\hline DIMENSIÓN - Conocimientos Profesionales & No Importante & Importante & $\mathbf{p}$ \\
\hline Evaluación Global - Conocimientos Profesionales $^{1}$ & & & 0,17 \\
\hline No asistió & $3(23,1 \%)$ & $10(76,9 \%)$ & \\
\hline Asistió & $5(8,9 \%)$ & $51(91,1 \%)$ & \\
\hline Total & $8(11,6 \%)$ & $61(88,4 \%)$ & \\
\hline Gestión y Legislación del Deporte $^{1}$ & & & $0,02^{*}$ \\
\hline No asistió & $6(46,2 \%)$ & $7(53,8 \%)$ & \\
\hline Asistió & $8(14,3 \%)$ & $48(85,7 \%)$ & \\
\hline Total & $14(20,3 \%)$ & $55(79,7 \%)$ & \\
\hline Biodinámica del Deporte ${ }^{2}$ & & & 0,69 \\
\hline No asistió & $1(7,7 \%)$ & $12(92,3 \%)$ & \\
\hline Asistió & $5(8,9 \%)$ & $51(91,1 \%)$ & \\
\hline Total & $6(8,7 \%)$ & $63(91,3 \%)$ & \\
\hline Psico-Socio-Cultural del Deporte ${ }^{2}$ & & & 0,39 \\
\hline No asistió & $2(15,4 \%)$ & $11(84,6 \%)$ & \\
\hline Asistió & $5(8,9 \%)$ & $51(91,1 \%)$ & \\
\hline Total & $7(10,1 \%)$ & $62(89,9 \%)$ & \\
\hline $\begin{array}{l}\text { Teoría y Metodología del Entrenamiento } \\
\text { Deportivo }^{2}\end{array}$ & & & 0,43 \\
\hline No asistió & $0(0 \%)$ & $13(100 \%)$ & \\
\hline Asistió & $4(7,1 \%)$ & $52(92,9 \%)$ & \\
\hline Total & $4(5,8 \%)$ & $65(94,2 \%)$ & \\
\hline DIMENSIÓN - Habilidades Profesionales & No Importante & Importante & $\mathbf{p}$ \\
\hline Evaluación Global - Habilidades Profesionales $^{2}$ & & & 0,39 \\
\hline No asistió & $2(15,4 \%)$ & $11(84,6 \%)$ & \\
\hline Asistió & $5(8,9 \%)$ & $51(91,1 \%)$ & \\
\hline Total & $7(10,1 \%)$ & $62(89,9 \%)$ & \\
\hline Planeamiento y Gestión Deportiva ${ }^{2}$ & & & 0,32 \\
\hline No asistió & $2(15,4 \%)$ & $11(84,6 \%)$ & \\
\hline Asistió & $4(7,1 \%)$ & $52(92,9 \%)$ & \\
\hline Total & $6(8,7 \%)$ & $63(91,3 \%)$ & \\
\hline Evaluación en Deporte $^{2}$ & & & 0,47 \\
\hline No asistió & $2(15,4 \%)$ & $11(84,6 \%)$ & \\
\hline Asistió & $6(10,7 \%)$ & $50(89,3 \%)$ & \\
\hline Total & $8(11,6 \%)$ & $61(88,4 \%)$ & \\
\hline Comunicación e Integración & & & 0,58 \\
\hline No asistió & $1(7,7 \%)$ & $12(92,3 \%)$ & \\
\hline Asistió & $3(5,4 \%)$ & $53(94,6 \%)$ & \\
\hline
\end{tabular}




\begin{tabular}{lrrr} 
Total & $4(5,8 \%)$ & $65(94,2 \%)$ & \\
\hline Autorreflexión y Actualización Profesional en & & & 0,28 \\
Deporte & & & \\
No asistió & $3(23,1 \%)$ & $10(76,9 \%)$ & \\
Asistió & $7(12,5 \%)$ & $49(87,5 \%)$ & \\
Total & $10(14,5 \%)$ & $59(85,5 \%)$ & \\
\hline EVALUACIÓN GLOBAL & No Importante & Importante & p \\
\hline Importancia Atribuida ${ }^{1}$ & & & 0,22 \\
No asistió & $3(23,1 \%)$ & $10(76,9 \%)$ & \\
Asistió & $6(10,7 \%)$ & $50(89,3 \%)$ & \\
Total & $9(13,0 \%)$ & $60(87,0 \%)$ & \\
\hline
\end{tabular}

Legenda: (1) Chi-cuadrado; (2) Exacto de Fisher; ( $\left.{ }^{*}\right)$ Diferencia Significativa.

Fuente: elaborada por los autores.

La importancia atribuida a los temas destacados en el constructo de competencia profesional y la ejecución de cursos de formación permanente mostraron asociación significativa únicamente con el indicador de Gestión y Legislación del Deporte $(p=0,02)$ (Tabla 2), evidenciándose que el grupo de entrenadores que asistieron a actividades de formación continua en los últimos años mostró índice más elevado de importancia atribuida $(85,7 \%)$ respecto a los entrenadores que no participaron (53,8\%).

Al comparar la relación existente entre la autopercepción de dominio y de importancia atribuida a la competencia profesional, se evidenció que en todos los indicadores, dimensiones y evaluación global del constructo de competencia profesional existieron diferencias significativas (valor de $p<0,010<0,05$ ) (Tabla 3). 
Tabla 3.

Relación entre autopercepción de dominio e importancia atribuida a las competencias profesionales de los entrenadores de tenis.

\begin{tabular}{|c|c|c|c|c|c|}
\hline $\begin{array}{l}\text { Competencias } \\
\text { Profesionales }\end{array}$ & $\begin{array}{c}\text { No domina } \\
\text { y no } \\
\text { considera } \\
\text { importante }\end{array}$ & $\begin{array}{c}\text { No domina } \\
y \\
\text { considera } \\
\text { importante }\end{array}$ & $\begin{array}{l}\text { Domina y } \\
\text { no } \\
\text { considera } \\
\text { importante }\end{array}$ & $\begin{array}{l}\text { Domina y } \\
\text { considera } \\
\text { importante }\end{array}$ & $\mathbf{P}$ \\
\hline \multicolumn{6}{|c|}{ DIMENSIÓN - Conocimientos Profesionales } \\
\hline $\begin{array}{l}\text { Evaluación Global - } \\
\text { Conocimientos Profesionales }\end{array}$ & $6(8,7 \%)$ & $23(33,3 \%)$ & $2(2,9 \%)$ & $38(55,1 \%)$ & $<0,01^{*}$ \\
\hline $\begin{array}{l}\text { Gestión y Legislación del } \\
\text { Deporte }\end{array}$ & $12(17,4 \%)$ & $31(44,9 \%)$ & $2(2,9 \%)$ & $24(34,8 \%)$ & $<0,01^{*}$ \\
\hline Biodinámica del Deporte & $5(7,2 \%)$ & $18(26,1 \%)$ & $1(1,4 \%)$ & $45(65,2 \%)$ & $<0,01^{*}$ \\
\hline $\begin{array}{l}\text { Aspectos Psico-Socio- } \\
\text { Culturales del Deporte }\end{array}$ & $3(4,3 \%)$ & $13(18,8 \%)$ & $4(5,8 \%)$ & $49(71,0 \%)$ & $0,05^{*}$ \\
\hline $\begin{array}{l}\text { Teoría y Metodología del } \\
\text { Entrenamiento Deportivo }\end{array}$ & $3(4,3 \%)$ & $9(13,0 \%)$ & $1(1,4 \%)$ & $56(81,2 \%)$ & $0,02^{*}$ \\
\hline \multicolumn{6}{|c|}{ DIMENSIÓN - Habilidades Profesionales } \\
\hline $\begin{array}{l}\text { Evaluación Global - } \\
\text { Habilidades Profesionales }\end{array}$ & $5(7,2 \%)$ & $20(29,0 \%)$ & $2(2,9 \%)$ & $42(60,9 \%)$ & $<0,01^{*}$ \\
\hline $\begin{array}{l}\text { Planeamiento y Gestión } \\
\text { Deportiva }\end{array}$ & $5(7,2 \%)$ & $15(21,7 \%)$ & $1(1,4 \%)$ & $48(69,6 \%)$ & $<0,01^{*}$ \\
\hline Evaluación en Deporte & $4(5,8 \%)$ & $22(31,9 \%)$ & $4(5,8 \%)$ & $39(56,5 \%)$ & $<0,01^{*}$ \\
\hline $\begin{array}{l}\text { Comunicación e Integración } \\
\text { en Deporte }\end{array}$ & $4(5,8 \%)$ & $14(20,3 \%)$ & $0(0 \%)$ & $51(73,9 \%)$ & $<0,01^{*}$ \\
\hline $\begin{array}{l}\text { Autorreflexión y } \\
\text { Actualización Profesional en } \\
\text { Deporte }\end{array}$ & $6(8,7 \%)$ & $16(23,2 \%)$ & $4(5,8 \%)$ & $43(62,3 \%)$ & $<0,01^{*}$ \\
\hline Evaluación Global & $8(11,8 \%)$ & $25(36,2 \%)$ & $1(1,4 \%)$ & $35(50,7 \%)$ & $<0,01^{*}$ \\
\hline
\end{tabular}

Legenda: Test de McNemar; $\left(^{*}\right)$ Diferencia Significativa.

Fuente: elaborada por los autores.

El índice de desinterés evidenciado por la muestra (Tabla 3), es decir, declarar que no se considera importante, pero domina los asuntos abordados en el constructo sobre la competencia profesional, fue bajo, con las puntuaciones de los indicadores, dimensiones y evaluación global llegando a un máximo de 5,8\%. Además, se destaca que el índice de concordancia entre el hecho de no dominar y no considerar importante los temas abordados en el constructo de competencia profesional, presentó un valor significativo solamente en el indicador Gestión y Legislación del Deporte $(17,4 \%)$. 
Por otro lado, el nivel de concordancia entre el hecho de dominar y considerar importante mostró gran variación en el constructo de competencia profesional (Tabla 3). Los indicadores: Aspectos Psico-Socio-Culturales del Deporte (71,0\%), Teoría y Metodología del Entrenamiento Deportivo $(81,2 \%)$ y Comunicación e Integración (73,9\%) presentaron los mayores índices de entrenadores que poseían dominio y daban importancia a estos asuntos. Por otro lado, únicamente el indicador Gestión y Legislación del Deporte mostraron bajo índice de entrenadores que poseían dominio y atribuían importancia a ese tema (34,8\%).

El porcentaje de entrenadores que se consideraban con bajo dominio, pero que entendían que los temas abordados en el constructo de competencia profesional son importantes varió entre $13,0 \%$ y $44,9 \%$. Se destaca que, al menos, una cuarta parte de la muestra reportó una necesidad de tener mayor dominio en la Evaluación Global de la Competencia Profesional, en las dimensiones Conocimientos y Habilidades Profesionales y en los indicadores Gestión y Legislación del Deporte, Evaluación en el Deporte, Biodinámica del Deporte. Sin embargo, el indicador del que los entrenadores indicaron mayor necesidad de dominio fue la Teoría y Metodología del Entrenamiento Deportivo.

\section{DISCUSIÓN}

El análisis general de los datos encontrados para la dimensión de Conocimiento Profesional demostró estar en concordancia con los resultados descritos por la literatura para entrenadores deportivos, en la que los conocimientos relacionados a las categorías Teoría y Metodología del Entrenamiento, a los Aspectos Psico-socioculturales del Deporte, y la Biodinámica, respectivamente en ese orden, muestran mayor autopercpeción de dominio (Egerland et al., 2010; Egerland, Salles, Barroso, Baldi y Do Nascimento, 2013; Egerland, et al., 2014). Al igual que se encontró esta similitud, diferencias substanciales fueron observadas en el porcentaje de entrenadores que indicaron dominar esos conocimientos, respecto a los entrenadores de la muestra que demostraron mayor criticidad sobre la autopercepción de competencia. Esta característica no parece ser una particularidad de los entrenadores de tenis, ya que ha sido observada en otros estudios con profesionales de otras modalidades individuales (Egerland et al., 2010; Egerland et al., 2014).

La verificación de que el $33 \%$ de la muestra afirmó no dominar los conocimientos relacionados con la categoría Biodinámica, revela la necesidad de poner mayor atención, por parte de las instituciones responsables de velar por el desarrollo de estos profesionales (universidades, confederaciones, federaciones, entre otras), y de un monitoreo constante de las necesidades y expectativas referentes a aprendizaje profesional. Los conocimientos envueltos 
en esta categoría son parte de la cotidianidad de los entrenadores de tenis, independientemente del contexto de actuación profesional (participación o rendimiento) (Egerland et al., 2009a). En ella se encuentran insertados los conocimientos relacionados al desarrollo y a las capacidades motoras, sobre nutrición, biomecánica y fisiología aplicada al deporte (Egerland et al., 2010).

En el ámbito internacional la capacitación de entrenadores se da por medio de tres vías distintas: la federativa (Federación Internacional de Tenis - ITF o por las confederaciones y federaciones; la universitaria o académica, ofrecida por las instituciones de enseñanza superior, y las formaciones impartidas por asociaciones privadas (Fuentes y Villar, 2004). Entre estas, las actividades de formación brindadas por la vía federativa son la más frecuentadas por los entrenadores de tenis en todo el mundo. En general, las titulaciones emitidas en éste ámbito se ofrecen divididas en tres o cuatro módulos, con cargas horarias, prerrequisitos y contenidos de cada módulo que van a variar de un país a otro (Fuentes y Villar, 2004).

Según Sanz, Fuentes e Villar (2004) las naciones en las que se va desarrollando esta modalidad, como es el caso de los países latinoamericanos, suelen elegir el modelo de capacitación establecido por la ITF. Este currículum con carácter generalista y compuesto por tres niveles de capacitación: un primer nivel enfocado en el trabajo con tenistas principiantes o con nivel intermedio de juego; un segundo nivel para tenistas avanzados, y un último nivel dirigido a los entrenadores interesados en trabajar con tenistas de alto rendimiento; donde los conocimientos profesionales requeridos con más frecuencia por los entrenadores son abordados con mayor profundidad a lo largo del proceso.

Particularmente, los conocimientos relacionados con la Biodinámica aparecen con frecuencia durante el proceso de capacitación (ITF, 2014). En este sentido, el no haber encontrado asociaciones estadísticamente significativas entre la participación en cursos/actividades de capacitación continua y la autopercepción de competencia para los conocimientos profesionales, refuerza la complejidad del proceso de aprendizaje, y promueve la necesidad de estudios que profundicen las especificidades de los entrenadores de tenis. Investigaciones recientes se han preguntado el impacto de las capacitaciones puntuales de corta duración en el aprendizaje profesional de los entrenadores deportivos (Trudel, Gilbert y Werthner, 2010). La forma superficial con la cual los conocimientos y competencias son abordados, las estrategias implementadas y la ausencia de un currículum estructurado que auxilie a los entrenadores en procesos de construcción de una identidad profesional innovadora, son algunos de los puntos criticables de este tipo de capacitaciones (Milistetd et al., 2016, Stoszkowski y Collins, 2016). 
Otro factor importante que aún necesita ser considerado. Las fuentes de aprendizaje de los entrenadores son muy variadas, lo que permite a los entrenadores no participar de actividades de capacitación continua, y desarrollarse por otros medios. Los entrenadores deportivos, en general, otorgan mayor significado a las situaciones no mediadas (Mesquita, Ribeiro, Santos y Morgan, 2014), ocurridas en el contexto informal (Stoszkowski y Collins, 2016; Walker, Thomas y Driska, 2018). En el caso específico de los entrenadores de tenis brasileños, la capacitación profesional se encuentra íntimamente ligada a la socialización previa como juntador de bolas (Cortela, Aburachid, Souza, Cortela y Fuentes García, 2013; Leite, Cieslak, Silva, Balbinotti y Cortela, 2016). Esa particularidad de la realidad nacional se aproxima, muchas veces, a la idea de "Escuela de Oficio" presentada por Drigo, Souza Neto, Cesana e Gomes Tojal (2011), en la que los conocimientos necesarios del ejercicio profesional son adquiridos por medio de experiencias vividas y con el contacto directo con un entrenador más experimentado. Tal comportamiento favorece el aparecimiento de oportunidades de aprendizaje ocurridas por medio de situaciones no mediadas y en el contexto informal.

En cuanto al dominio de las habilidades, los resultados encontrados son conformes con los demostrados en literatura previa, con las habilidades de Planeamiento y Gestión Deportiva, Comunicación e Integración, Evaluación en el Deporte, y Autorreflexión y Actualización Profesional, en ese orden, siendo relatadas como las de mayor dominio (Egerland, Nascimento y Both, 2009b; Egerland et al., 2013; Egerland et al., 2014). Así como lo observado para los conocimientos profesionales, los entrenadores de tenis de la muestra se mostraron más críticos con relación a la percepción de dominio, lo que refuerza la necesidad de desarrollar estudios contextualizaos para la elaboración de acciones de capacitación permanente.

La participación en actividades de capacitación permanente mostró ser eficaz en la autopercepción de dominio para la dimensión Habilidades Profesionales y en los indicadores Planeamiento y Gestión Deportiva y Evaluación en el Deporte. Según el Instituto Portugués de Deporte y Juventud (2016) la actividad del entrenador ocurre en un ciclo ininterrumpido de planear, aplicar y evaluar sus sesiones de entreno. Esas habilidades están encuadradas en el rol de competencias centrales para el ejercicio de los entrenadores establecidos por la ICCE (2013), siendo considerada pre-requisito para llegar a los más elevados dominios como profesional. En este sentido, se observa que la participación en actividades de capacitación continua se muestra como una importante oportunidad de aprendizaje, pudiendo auxiliar a los entrenadores de tenis en el desarrollo de habilidades fundamentales para el desempeño de sus funciones. 
De manera semejante a lo demostrado en otros estudios (Egerland et al., 2009a; Egerland et al., 2009b; Egerland et al., 2013) se observó que la importancia atribuida a los conocimientos y habilidades profesionales fue elevada. En esa misma dirección, se verificó que la importancia atribuida fue siempre superior al dominio que se poseía, señalando lagunas y puntos de mejoría para el desarrollo de acciones de capacitación permanente. Los conocimientos profesionales relativos a la categoría Gestión y Legislación Deportiva recibieron una menor atribución de importancia. Esto puede estar asociado a la falta de dominio del componente (Tablas 1 e 2 ), y/o menor importancia de este conocimiento para el contexto de actuación profesional del grupo en cuestión.

Asociaciones estadísticamente significativas fueron encontradas entre la autopercepción de importancia sobre los conocimientos de Gestión y Legislación Deportiva y la participación en actividades de capación permanente, indicando que los entrenadores que participan en ese tipo de capacitación tienden a atribuir mayor valorización. En esta categoría se encuentran insertos los saberes relacionados con la Gestión y Organización, Legislación básica, Doping y Funciones directivas (Egerland et al., 2010). A mayor importancia de estos profesionales en continuar desarrollándose en diferentes áreas del conocimiento. De la misma manera que para este grupo, la autopercepción de dominio mostró estar por debajo de lo esperado, verificándose que menos de la mitad de los profesionales indicaron dominar esos conocimientos. El análisis curricular de las capacitaciones más frecuentadas por lo entrenadores permite afirmar que las temáticas ligadas a la gestión y legislación no son parte del alcance del programa (Confederación Brasileña de Tenis [CBT], 2017), posición basada en los resultados observados.

Los datos mostrados en la Tabla 3 señalan el cuadro general sobre las competencias y necesidades de los entrenadores de tenis, indicando posibilidades de avances para la reestructuración de programas de capacitación continua que puedan atender de mejor forma a estos profesionales. Dentro de los conocimientos profesionales, aquellos relacionados con la Gestión y Legislación Deportiva y, Biodinámica del Deporte fueron identificados como puntos que deben tratarse dentro de los programas, debido a la cantidad de entrenadores que expresaron no dominar estas temáticas, pero juzgaron como importantes esas áreas de conocimiento. En esta misma dirección, los resultados observados para las habilidades de Evaluación Deportiva también requieren mayor atención, debido a la importancia de esta habilidad para el ejercicio profesional de entrenador de tenis (Instituto Portugués de Deporte y Juventud, 2016; ICCE, 2013). 


\section{Consideraciones Finales}

En conclusión, se observa la necesidad de mirar nuevamente el impacto de las actividades específicas de capacitación continua en la autopercepción de competencia profesional de los entrenadores de tenis. Respondiendo a los objetivos propuestos para el estudio, los resultados encontrados demuestran que la participación en cursos/actividades de formación permanente, desarrollados en el contexto no formal, no demostró asociación significativa con la autopercepción de competencia de estos entrenadores, con respecto a los Conocimientos Profesionales. Por otro lado, esas capacitaciones demostraron impactar la autopercepción de dominio en la dimensión Habilidades Profesionales y para las categorías Planeamiento/Gestión Deportiva y Evaluación Deportiva, dos habilidades consideradas centrales en el ejercicio profesional den entrenador.

La importancia atribuida a los conocimientos y habilidades profesionales, de modo general, fue elevada, con los entrenadores que participaron de capacitaciones atribuyendo mayor importancia a los conocimientos relacionados a la Gestión y Legislación Deportiva. Esta categoría de conocimiento fue la que presentó los mayores contrastes en relación a la autopercepción de dominio e importancia atribuida. A su vez, las habilidades relacionadas con la Evaluación en el Deporte demostraron ser las más deseadas por los entrenadores que no las dominaban, pero que le atribuían importancia a ese indicador.

La verificación de que la participación en cursos no demostró asociación significativa con la autopercepción de dominio para los Conocimientos Profesionales, refuerza el carácter individual del aprendizaje y la necesidad de estudios contextualizaos para intervenciones ms asertivas en el desarrollo profesional de los entrenadores. Por ser considerados los conocimientos especializados del coaching deportivo, esos conocimientos han sido basados en los programas de capacitación de entrenadores, lo que requiere la ejecución de un análisis detallado de los currículum y de las estrategias de enseñanza en estas actividades de capacitación permanente.

\section{AGRADECIMIENTOS}

Los autores agradecen a los entrenadores que participaron en el estudio y al apoyo ofrecido por la Federación Paranaense de Tenis. 


\section{REFERENCIAS}

Ciampolini, V., Milistetd, M., Rynne, S., Brasil, V.Z. y Nascimento, J.V. (2019). Research review on coaches' perceptions regarding the teaching strategies experienced in coach education programs. International Journal of Sports Science \& Coaching, 14(2), 216228. doi: https://doi.org/10.1177/1747954119833597

Confederação Brasileira de Tênis [CBT]. (2017). Estrutura dos Cursos de formação oferecidos pelo Departamento de Capacitação. Recuperado de $\underline{\text { http://cbt- }}$ tenis.com.br/capacitacao.php?cod=3

Cortela, C.C., Aburachid, L.M., Souza, S.P., Cortela, D.N.R. y Fuentes García, J.P. (2013). A formação inicial e continuada dos treinadores paranaenses de tênis. Conexões: Educação Física, Esporte e Saúde, 11(2), 60-84. doi: https://doi.org/10.20396/conex.v11i2.8637617

Drigo, A.J., Souza Neto, S., Cesana, J. y Gomes Tojal, J.B.A. (2011). Artes marciais, formação profissional e escolas de ofício: Análise documental do judô brasileiro. Motricidade, 7(4), 49-62. Recuperado de https://bit.ly/2Nwp9kS

Duffy, P., Petrovic, L. y Crespo, M. (2010). The European framework for the recognition of coaching competence and qualifications - implications for the sport of athletics in Europe: a report to European Athletics. New Studies in Athletics, 25(1), 27-41. Recuperado

de https://www.iaaf.org/nsa/article/filter?\&articleTitle=the\%20european\%20framework\&yea $r=2010$

Egerland, E.M., Do Nascimento, J.V. y Both, J. (2009a). Professional competences of sports couches of santa catarina state. Motriz. Journal of Sports of Physical Education, 15(4), 890-899.

Recuperado

de http://www.periodicos.rc.biblioteca.unesp.br/index.php/motriz/article/view/2946

Egerland, E.M., Nascimento, J.V. y Both, J. (2009b). Nível de associação entre a importância atribuída e a competência percebida de treinadores esportivos. Pensar a Prática, 12(3), 1-13. doi: https://doi.org/10.5216/rpp.v12i3.7621

Egerland, E.M., Nascimento, J.V. y Both, J. (2010). Competência profissional percebida de treinadores esportivos catarinenses. Journal of Physical Education, 21(3), 457-467. doi: https://doi.org/10.4025/reveducfis.v21i3.8285

Egerland, E.M., Salles, W.D.N., Barroso, M.L.C., Baldi, M.F. y Do Nascimento, J.V. (2013). Potencialidades e necessidades profissionais na formação de treinadores desportivos. 
Revista Brasileira de Ciência e Movimento, 21(2), 31-38. doi: http://dx.doi.org/10.18511/0103-1716/rbcm.v21n2p31-38

Egerland, E., Salles, W.N. y Baldi, M.F. (2014). Perception of professional competence of Brazilian college coaches. Revista Brasileira de Cineantropometria \& Desempenho Humano, 16(4), 437-446. doi: http://dx.doi.org/10.5007/1980-0037.2014v16n4p437

Fuentes, J.P.G. y Villar, F.A. (2004). El entrenador de tenis de alto rendimento: un estudio sobre su formación inicial y permanente. Badajoz: APROSUBA -3.

Governo do Brasil (1998). Lei 9.696, 1 de setembro de 1998. Recuperado de http://www.planalto.gov.br/ccivil 03/leis/19696.htm

Instituto Portugués do Desporto e Juventude. (2016). Didática do desporto. In O. Coelho. Plano nacional de formação de treinadores: manuais de formação Grau I (pp. 1-27). Lisboa: Instituto Português de Desporto e Juventude.

International Council for Coaching Excellence [ICCE]. (2013). International Sport Coaching Framework Version 1.2. Illinois: Human Kinetics; Champaign. Recuperado de https://www.icce.ws/ assets/files/iscf-1.2-10-7-15.pdf

International Tennis Federation [ITF]. (2014). Coach education programme. Recuperado de http://en.coaching.itftennis.com/coach-education/coach-education-programme.aspx

Leite, E.V., Cieslak, F., Silva, C.F., Balbinotti, C.A.A. y Cortela, C.C. (2016). Qualidade de vida, percepção de imagem corporal e aspectos de sonolência em treinadores de tênis de Campo Grande. Revista Brasileira de Qualidade de Vida, 8(2), 175-190. doi: https://doi.org/10.3895/rbqv.v8n2.3881

Lemos, C.A.F. (2007). Qualidade de vida da carreira profissional de professores de Educação Física do magistério público estadual/Rs (Dissertação de mestrado). Universidade Federal de Santa Catarina, Programa de Pós-graduação em Educação Física, Florianópolis, Brasil. Recuperada de http://repositorio.ufsc.br/xmlui/handle/123456789/89948

Mesquita, I., Ribeiro, J., Santos, S. y Morgan, K. (2014). Coach, learning and coach education: A Portuguese expert coaches' perspective. The Sport Psychologist, 28(2), 124-136. doi: https://doi.org/10.1123/tsp.2011-0117

Milistetd, M., Ciampolini, V., Salles, W.N., Ramos, V., Galatti, L.R. y Do Nascimento, J.V. (2016). A. Coaches' development in Brazil: structure of sports organizational programs. Sports Coaching Review, 5(2), 1-13. doi: https://doi.org/10.1080/21640629.2016.1201356 
Milistetd, M., Galatti, L.R, Tozetto, A., Collet, C. y Do Nascimento, J.V. (2017). Formação de treinadores esportivos: orientações para a organização das práticas pedagógicas nos cursos de bacharelado em educação física, Journal of Physical Education, 28, 1-14. doi: https://doi.org/10.4025/jphyseduc.v28i1.2849

Rodrigues, H.A., Paes, R.R. y Souza Neto, S. (2016). A socialização profissional do treinador esportivo como um processo formativo de aquisição de saberes. Movimento. Revista de Educação Física da UGRGS, 22(2), 509-521. doi: https:/doi.org/10.22456/1982$\underline{8918.55346}$

Sanz, D.R., Fuentes, J.P. y Villar, F.A. (2004). La formación del entrenador de tenis. In torres, G., Carrasco, L. (Eds.). Investigación en deportes de raqueta: tenis y bádminton (pp: 341-364). Múrcia: Universidad Católica San Antonio editora.

Silva, S.G., Minatto, G., Fares, D. y Santos, S.G. (2011). Caracterização da Pesquisa (Tipos de Pesquisa). In S.G. Santos (Ed.), Métodos e Técnicas de Pesquisa Quantitativa Aplicada à Educação Física (pp. 67-73). Florianópolis: Tribo da llha.

Stoszkowski, J. y Collins, D. (2016). Sources, topics and use of knowledge by coaches. Journal of Sports Sciences, 34(9), 794-802. doi: https://doi.org/10.1080/02640414.2015.1072279

Taylor, W. y Garrat, D. (2013). Coaching and professionalization. In P. Potrac, W. Gilbert e J. Denison (Eds.), Routledge Handbook of Sports Coaching (pp. 27-39). London: Routledge.

Trudel, P. y Gilbert, W. (2013). The role of deliberate practice in becoming an expert coach: Part 3 - Creating optimal settings. Olympic Coach Magazine, 24(2), 15-28. Recuperado de http://www.teamusa.org/About-the-USOC/Athlete-Development/CoachingEducation/Coach-E-Magazine

Trudel, P., Gilbert, W. y Werthner, P. (2010). Coach Education Effectivenes. In: J. Lyle \& C.J. Cushion (Eds.). Sports Coaching: Professionalization and Practice (pp. 135-152). London: Elsevier.

Walker, L.F., Thomas, R. y Driska, A.P. (2018). Informal and nonformal learning for sport coaches: A systematic review. International Journal of Sports Science \& Coaching, 13(5), 694-707. doi: https://doi.org/10.1177/1747954118791522 